\title{
Musik mit dem Radio hören: Über den Begriff der musikalischen Aufführung
}

\author{
MAGDALENA ZORN, MÜNCHEN
}

Bis heute beobachten Vertreter*innen von Aufführungsdisziplinen wie den Theater-, Film- und Tanzwissenschaften die Diskurse der historischen Musikwissenschaft aufgrund der ihnen inhärenten Medienvergessenheit bisweilen skeptisch. Anlass zur Kritik bietet den Skeptiker*innen nicht nur die raumgreifende musikwissenschaftliche Schriftversessenheit in der Erforschung von klingenden Kunstwerken, sondern auch eine ganz spezifische Auffassung von musikalischer Aufführung. So herrscht in weiten Teilen der internationalen Musikwissenschaft noch immer der Konsens vor, dass die einzige Aufführung, die ihren Begriff voll und ganz erfüllt, die sogenannte Live-Aufführung von Musik in Konzertsälen, Opernhäusern, Kreativquartieren und öffentlichen Räumen darstellt. Mit den technisch-medialen Revolutionen des 20. und 21. Jahrhunderts wurde Musik jedoch, auch diejenige, die in „Werken" vorliegt, für den Großteil der Menschen eine technisch "vermittelte Kunst". Selbst einer Beethoven-Symphonie haben sich dabei Signaturen von technisch-medialen Diskursapparaturen eingeschrieben, die musikwissenschaftliche Analyse nicht mehr ignorieren kann. Beethovens Kompositionen, in denen die Philosophin Lydia Goehr den Inbegriff einer Verwirklichung des europäischen WerkKonzeptes sah, ${ }^{1}$ haben in der Rezeptionssituation des Radios, die vom heimischen Wohnzimmer bis hin zum Mobile Listening mit iPod die unterschiedlichsten Gebrauchskontexte verschränkt, ihren Status als autonome Kunstwerke verloren. Sowohl ihre mediatisierte Reproduktion und Verbreitung als auch ihre mediale Rezeption - vom Konsum bis hin zur Reflexion - betten die Musik dabei in heteronome Kontexte ein, die die Vorstellung von einer der Welt des Alltags gänzlich enthobenen musikalischen Aufführungssituation als überkommen erscheinen lassen. Dass eine Annäherung an den Aufführungsbegriff für durch das Radio mediatisierte Musik alles andere als leicht gelingt, hat wesentlich mit den Bildern unserer Sprache zu tun, die uns in diesem Fall "gefangen"2 halten und die Sackgasse der Medienvergessenheit weiter zementieren. So sprechen wir für gewöhnlich davon, dass wir Musik "im" Radio zuhören und suggerieren damit implizit unser Einverständnis darüber, dass das Radio ein bloßes Medium der Musikübertragung sei (Abb. 1). Die Setzung der Präposition „im” beinhaltet die Annahme, dass wir es bei einer Radioübertragung mit einer Musikvermittlung zu tun haben, die das musikalische Original erhält.

1 Vgl. Lydia Goehr, The Imaginary Museum of Musical Works. An Essay in the Philosophy of Music, Oxford 22007, S. 205-242.

2 "Ein Bild hielt uns gefangen. Und heraus konnten wir nicht, denn es lag in unserer Sprache, und sie schien es uns nur unerbittlich zu wiederholen". Ludwig Wittgenstein, Philosophische Untersuchungen. Kritisch-Genetische Ausgabe, hrsg. von Joachim Schulte et. al., Frankfurt a.M. 2001, § 115. Hervorhebung im Original. 


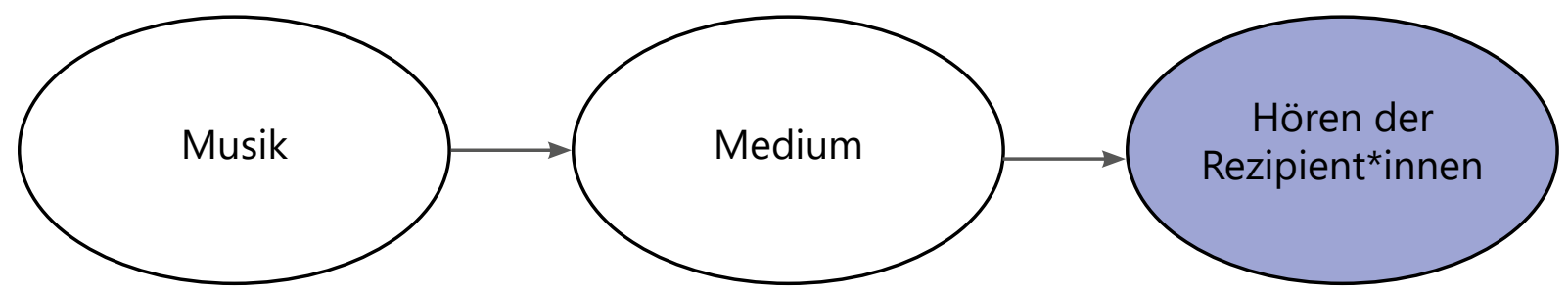

Abbildung 1: Das Radio als Medium der Musik

Diese Kommunikationsstruktur wird jedoch sogleich unglaubwürdig, wenn wir uns vergegenwärtigen, dass das Radio selbstverständlich kein bloßes Übertragungsmedium ist. Denn jede mit der Übertragung zur Live-Aufführung hinzukommende ästhetische Qualität ist „Zeichen der Integrität" ${ }^{\prime 3}$ des Hörens des Radios selbst. Nehmen wir den Fall einer Radioübertragung einer Symphonie aus dem Konzertsaal. Die technischen Möglichkeiten des Soundengineerings bei großen Radiosendern sind mittlerweile so weit fortgeschritten, dass dieses musikalische Werk sogar bei einer Live-Übertragung mehr oder minder tiefgreifende Veränderungen erfährt: Nachhallzeiten werden verkürzt und die dynamischen Verhältnisse zwischen den Instrumenten nach Maßgabe der Partitur reguliert. Wer jemals den Radiomitschnitt einer musikalischen Aufführung gehört hat, bei der er selbst im Konzert live zugegen war und dann die Diskrepanz zwischen diesen beiden Hörerfahrungen bemerkt, hat am eigenen Leib erfahren, dass das Radio selbst Ohren hat. Es lässt sich also sagen, dass das Medium Radio der musikalischen Aufführung Spuren eines Hörens einschreibt, noch bevor Rezipient*innen diese überhaupt gehört haben. Das Radio schaltet zwischen die Aufführung eines musikalischen Werkes und unser Hören als Rezipient*innen das "Hören des Radios" (Abb. 2). Der Genitivus subiectivus bringt das auditive Tätigsein des medialen Apparates selbst zum Ausdruck.

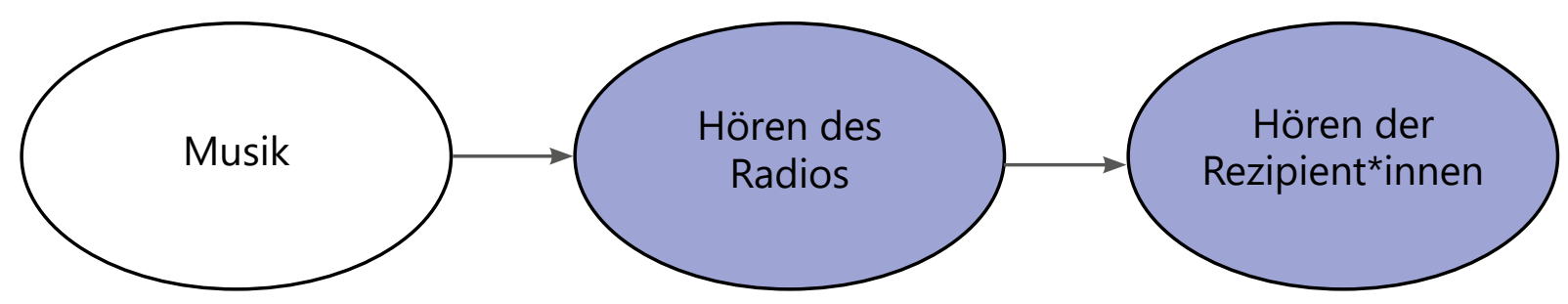

Abbildung 2: Das Radio als Hören der Musik

Gewiss, wir machen uns das auditive Tätigsein des Radios bei einer Übertragung aus dem Konzertsaal im Normalfall während der Musikwahrnehmung nicht bewusst und richten unsere Aufmerksamkeit an Stelle dessen auf die Musik, die wir an einem Ort "hinter" dem Radio ver-

3 Arthur C. Danto, Die Verklärung des Gewöhnlichen: Eine Philosophie der Kunst (The Transfiguration of the Commonplace: A Philosophy of Art, 1981), Frankfurt a.M. 1991, S. 169. 
muten. Wir gehen dabei wie selbstverständlich von der Annahme aus, dass das Medium Radio transparent ist und uns durchsichtig auf die Musik selbst verweisen könnte. Ganz selten ereignen sich solche Fälle, in denen sich das Hören des Radios für uns viel lauter zu Wort meldet als die übertragene Musik selbst. Derlei Situationen treten etwa dann ein, wenn Störgeräusche in der Übertragung zu hören sind. Das Hören des Radios produziert dann störende Hörgeräusche und alle hören für einen Moment plötzlich dem Radio bei seinem Hören zu. Indes macht das Hören des Radios permanent auch ästhetische Geräusche, die wir aber geneigt sind, zu ignorieren. So hört das Radio musikalische Nähe und Distanz, die klanglichen Relationen aus Vordergrund und Hintergrund, komponierte Räumlichkeit und Zeitlichkeit, aber wir schreiben das vielfach der Musik zu, die "im" Radio erklingt, obwohl sie in Wahrheit "mit dem Radio" als Medium miterklingt und dabei von allem Anfang an etwas anderes war als nur Musik, nämlich vom Radio bereits gehörte Musik. Diese Idee der Radiomusik bereitet uns Probleme, denn sie transformiert unsere Auffassung von einem Medium tiefgreifend: Das Radio erscheint dabei nicht nur als Medium für "unser" Hören, sondern auch als ein Medium, indem sich unsere auditive Wahrnehmung spiegelt. In gewissem Sinne könnte man sogar sagen, das Radio und wir sind dasselbe, nämlich Medien des Hörens.

Die kommunikative Funktion des Radios liegt in zwei Sprachhandlungen: in seinem indikativischen Bezug auf musikalische Ereignisse, die es überträgt („Ich höre eine BeethovenSymphonie"), und in seiner imperativischen Adressierung an uns Hörer*innen von Radiomusik. Das Radio fordert uns auf, es ihm gleichzutun und zu hören, es adressiert an uns den Imperativ: „Hör' mir zu!". Und wir erfüllen diesen Imperativ nur dann, wenn wir nicht nur der Musik, sondern auch dem Radio beim Hören zuhören: wenn wir eben Beethovens Musik "mit dem" Radio hören. Ich folge in dieser Ansicht, wonach das Hören des Radios zugleich eine indikativische Aussage über das Gehörte und einen Imperativ an Hörer*innen darstellt, dem französischen Philosophen Peter Szendy, der die Entdeckung machte, dass das französische Lexem „écoute" zugleich Nomen für das auditive Tätigsein einer Instanz - in diesem Fall das Radio - und Aufforderung zur Audition - in diesem Fall an uns Radiohörer*innen - ist. ${ }^{4}$

Das Radio enthält also ganz bestimmte Angebotsbestimmungen, der Musik Beethovens Gehör zu schenken. Hören wir der Beethoven-Symphonie "mit dem" Radio zu, finden wir uns auf die ästhetische und soziologische Affordanz eines technischen Mediums verwiesen, das den Sinn der übertragenen Musik nachhaltig verändert. Von der Beethoven-Symphonie geht nun plötzlich nicht mehr nur das Angebot zur konzentrierten und von jeder Ablenkung befreiten Audition aus, wie es das Dispositiv des Musikhörens im modernen Konzert besagt, sondern vielmehr die Aufforderung, das musikalische Ereignis in das privatistische Leben des Alltags zu integrieren, um nicht zuletzt auch gänzlich subjektive emotionale und intellektuelle

4 Vgl. Peter Szendy, "Otographes”, in: Circuit: musiques contemporaines 13, H. 2 (2003), S. 11-26, hier S. 13. Vgl. dazu Peter Szendy, Höre(n). Eine Geschichte unserer Ohren (Écoute. Une histoire de nos oreilles, 2001), übers. von Daniel Schierke, Paderborn 2015. 
Bedürfnisse zu befriedigen. Welche Konsequenzen hat dieser technisch-mediale Gebrauch des klassischen Werkes für die musikwissenschaftliche Analyse? Deutlich wird in jedem Fall, dass sich eine solche Analyse weder ausschließlich auf die Partitur des Werkes noch auf die LiveAufführung beziehen kann, sondern in umfassendem Sinne nach den soziologischen Rahmenbedingungen musikalisch-ästhetischer Erfahrung von Subjekten in Kontexten technischmedialer Musikrezeption fragen muss.

Die Sprache über Musik verdeckt die mediale Eigenheit des Radiohörens auch noch in einer anderen Hinsicht. Die Limitierungen des Sprechens zeigen sich zusätzlich im Blick auf die unterschiedlichen Gebrauchsformen der deutschen Verben "hören" und "zuhören". Jede auditive Erfahrung setzt sich aus "hören" und "zuhören" zusammen. Während das Subjekt beim "Hören" tendenziell affektiv in Bezug zu einem akustischen Ereignis tritt, entspricht das "Zuhören" einem kognitiven Prozess der Informationsverarbeitung. Die affektive Zuständlichkeit des "sens sensible" steht in auditiven Erfahrungen stets der kognitiven Prozessualität des "sens sensé" gegenüber, der das Hörbare dekodieren will. ${ }^{5}$ Beide Modi von Audition sind im Fall von Musikerfahrung wechselseitig voneinander abhängig: Das Subjekt braucht die affektive Bindung zur Musik, um ihr überhaupt in einem mentalen Verarbeitungsprozess folgen zu können, und umgekehrt entfacht die intellektuelle Erkenntnis erst eine affektive Beziehung zur Musik. Für den Gegenstand der Radiomusik nun bringen die Usancen dieser deutschen Hörverben einen interessanten Befund: Die Aussage "Ich höre das Radio der Nachbarin" ist ohne Weiteres möglich. Sie besagt schlichtweg, dass das hörende Subjekt akustisch das Radio der Nachbarin vernimmt. Der Satz "Ich höre dem Radio zu", stellt im Deutschen allerdings keine gängige Sprachhandlung dar. Er würde bedeuten, dass sich das sprechende Subjekt kognitiv auf die auditiven Informationen des Radios selbst ausrichtet. Der Satz ist pragmatisch nicht wohlgeformt und wird es erst dann, wenn wir ergänzen: „Ich höre dem Radio zu, aus dem Musik erklingt", oder noch besser: "Ich höre der Musik zu, die aus dem Radio erklingt". Dass die Formulierung "das Radio hören" gebräuchlich ist, die Redeweise "dem Radio zuhören" aber ungelenk wirkt, hat einen bestimmten Grund. Es gibt eine Reihe pragmatischer Evidenzen, die zeigen, dass wir sowohl Subjekte als auch Objekte "hören" können, demgegenüber aber eher nur demjenigen „zuhören”, von dem wir annehmen, dass es belebt ist, uns etwas zu sagen hat und vor allen Dingen, dass es mit uns kommunizieren will. ${ }^{6}$ Da wir dem Radio im Normalfall keine Belebtheit und keine kommunikative Agentivität unterstellen, sondern nur der Musik, die mit dem Radio miterklingt, ist das Radio selten explizit das Objekt unseres "Zuhörens", mit dem wir seiner medialen Beschaffenheit kognitiv auf den Grund gehen würden. Bis in die Grundfesten des deutschen Sprachgebrauchs hinein zeigt sich also im Sprechen über Radiomusik

5 Der französische Philosoph Jean-Luc Nancy charakterisierte Audition durch solche Doppeltheit von "sens sensible" und "sens sensé". Vgl. Jean-Luc Nancy, À l'écoute, Paris 2002, S. 18.

6 Vgl. Ewa Trutkowski, „Hören versus Zuhören: Dativ-Kasus als Marker für Agentivität”, in: (Zu-)Hören interdisziplinär, hrsg. von Magdalena Zorn und Ursula Lenker (= Münchner Veröffentlichungen zur Musikgeschichte, Sonderband 1), München 2018, S. 73-88. 
eine gewisse Medienvergessenheit. Die Sprache macht uns vergessen, dass das Radio selbst ein mit der Musik und uns kommunizierender (Zu-)Hörer ist, und lässt uns in den Wissenschaften dessen Imperativ "Hör' mir zu!" vielfach überhören.

Theodor W. Adorno war einer der ersten Musikphilosophen, der tatsächlich dem Radio beim Hören zuhörte. Die während seines New York-Aufenthaltes von 1938 bis 1941 fragmentarisch verschriftlichten Aufsätze, die nachträglich unter dem Titel Current of Music: Elements of a Radio Theory erschienen sind, erkunden die Frage, wie das Radio die Wahrnehmung von Musik transformiert. Adornos Thesen sind dabei offenkundig von der Gewissheit getragen, dass das Ohr des Radios bedingungslosen Gehorsam seiner Adressat*innen verlange und überdies derart laute Hörgeräusche produziere, dass die Integrität der großen europäischen Musik, wenn sie Gegenstand der Übertragung ist, davon nachhaltig beschädigt würde. Im Aufsatz "The Radio Symphony: An Experiment in Theory" geht der Musikphilosoph und -soziologe vom Beispiel einer Radioübertragung einer Beethoven-Symphonie aus. Eine Radioausstrahlung von Beethovens Musik ermögliche zwar vordergründig, so Adorno, dass nun auch die "Frauen der Farmer" ${ }^{17}$ im Mittleren Westen einen Zugang zur Great Music erhalten, doch in Wahrheit werde den Hörer*innen nur mehr ein Abklatsch des originären Werkes präsentiert, zu aufdringlich sei das Hören des Radios selbst. Vor allem zwei Aspekte kritisierte Adorno an der Radioübertragung einer Beethoven-Symphonie. Erstens sei die Symphonie durch das eklektizistische Mediendispositiv nicht mehr in ihrer zeitlichen Erstreckung zugänglich, was zur Trivialisierung der Musik selbst führe:

"The trivialization of symphony, first of all, is bound up with its relapse into time [...] The time the radio symphony consumes is empirical time. It is in ironic keeping with the technical limitations imposed by radio on the live symphony that they are accompanied by the listener's capacity to turn off the music whenever he pleases. He can arbitrarily supersede it in contrast to the concert hall performance where he is forced, as it where, to obey its law." ${ }^{8}$

Adorno ging äußerst unzureichend davon aus, dass das Konzert ganz im Unterschied zur radiofonen Situation eine vom Alltag der Hörenden vollkommen unbeeindruckte ästhetische Welt für sich selbst darstelle. Auch im Konzert jedoch machen Subjekte keine ungeteilte Erfahrung. Sie sind durch ihr eigenes Husten, die innige Miene des Konzertmeisters oder den Ausschnitt der Solistin abgelenkt und tragen dabei immer die Welt in die Erfahrung von Musik hinein. Die abschweifende Erfahrung charakterisiert jede realistische Situation des Musikhörens, weil es Menschen sind, die hören, und dieses Menschsein in ganz spezifischen historischen und soziologischen Kontexten jenseits und doch zugleich in der Kunst trägt bis heute maßgeblich zum Fortleben der Beethoven-Symphonien bei. Der zweite übergeordnete Kritikpunkt Adornos betrifft die ",surrounding' quality of music", ihre das Subjekt immersiv involvierende klang-

7 Vgl. Theodor W. Adorno, "The Radio Symphony: An Experiment in Theory" (1941), in: Current of Music: Elements of a Radio Theory, hrsg. von Robert Hullot-Kentor, Cambridge 2009, S. 144-162, hier S. 145.

8 Ebd., S. 155.

9 Ebd., S. 151. 
liche Ausbreitung, die dem Theoretiker im Radio zu kurz kam. Die Musik, so Adorno, sei ein Medium, in dem man leben müsse, und klar ist, dass in seiner Sichtweise einzig die Live-Aufführung dieses Leben-im-Klang ermöglichen konnte: „To ,enter' a symphony means to listen to it not only as something before one, but as something around one as well, as a medium in which one ,lives'." 10

Auffallend an Adornos Radiomusik-Kritik, die daneben auch mit einer Invektive gegen das "atomistic listening" ${ }^{\prime 11}$ und das "quotation listening"12 der Radiohörer*innen aufwartet, ist eine fundamentale Schieflage in der Argumentation. So machte der Musikphilosoph für das musikalische Werk zwar grundsätzlich einen Aufführungsbegriff stark, indem er die musikalischästhetische Erfahrung an das Erleben der Live-Situation im Konzert knüpfte, dementierte diesen musikalischen Aufführungsbegriff jedoch im Fall von Radiomusik wieder zur Gänze.

Dabei stellt sein Aufsatz, jenseits seiner kulturkonservativen Valenzen, eigentlich ein gedankliches Instrumentarium zur Verfügung, das den Aufführungscharakter von technischmedial vermittelter Musik auf der funktionalen Ebene einsichtig machen könnte. Adorno ging im Aufsatz ganz offensichtlich von der zentralen Hypothese aus, dass Radiohörer*innen, indem sie die zeitliche und räumliche Erstreckung einer Beethoven-Symphonie negieren, nicht mehr „auf die richtige Weise" zuhören. Daraus ergibt sich in letzter Konsequenz der Schluss, dass das Hören des Radios und das Hören der Rezipient*innen das Werk Beethovens nachhaltig transformieren. Mit dieser Auffassung wird für die Kommunikationsstruktur einer Radioübertragung von Musik eine neuerliche Korrektur erforderlich. Aus der Perspektive des von Adorno ausformulierten Aufführungsbegriffes des Radios vertauschen sich die Kommunikationsvektoren sogar. Wesentlich für die von ihm beschriebene Verhandlungssituation der Radiomusik ist nämlich das Faktum, dass Rezipient*innen in wechselseitiger Vermittlung mit dem Dispositiv Radio, von dem ein ebenso technischer, wie soziologischer und schließlich auch ästhetischer Angebotscharakter zum Hören ausgeht, ein musikalisches Ereignis adressieren. Der wichtigste Aufführungsparameter von Radiomusik, dass eben das Hören in der empirischen Zeit und im empirischen Raum des privatistischen Alltagslebens stattfindet, schreibt dem ästhetischen musikalischen Ereignis erst seine von der Live-Aufführung abweichenden Gehalte ein (Abbildung 3).

\footnotetext{
10 Ebd., S. 150.

11 Vgl. ebd., S. 155.

12 Vgl. ebd., S. 156-158.
} 


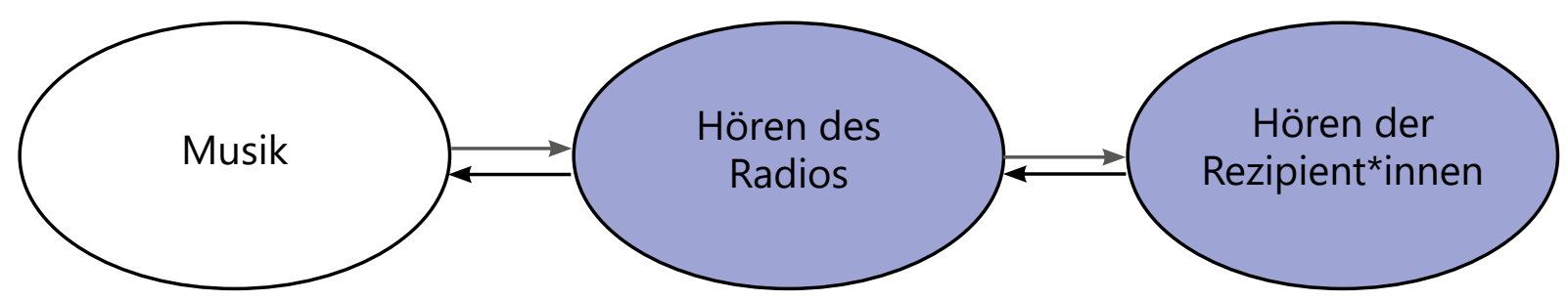

Abbildung 3: Adressierung der Musik durch das Hören

Implizit formulierte Adorno damit am Beispiel von Radiomusik einen Befund, der für den musikalischen Aufführungsbegriff insgesamt konstitutiv ist: dass nämlich jedes Medium des Hörens aus dem Werk ein neues Werk macht. In jeder musikalischen Aufführung entsteht Musik als Resultat einer von Rezipient*innen ausgehenden Erzeugungsweise von Sinn. In der Konterlektüre von Adornos Radio-Aufsatz zeigt sich, dass ausgerechnet das Hören mit dem Radio phänomenologische Gehalte offenbart, die für das musikalische Werk als Aufführung insgesamt entscheidend sind. Es scheint, als ob das Radio dasjenige Medium wäre, das uns nicht nur eine Beethoven-Symphonie vermittelt, sondern uns sogar das Phänomen musikalisch-ästhetischer Wahrnehmung erklären kann. Indem das Radio, mit Adorno gesprochen, die präsentischen Qualitäten der Konzert-Aufführung unterschlägt, stößt es uns ex negativo erst auf all jene ästhetischen Gehalte der musikalischen Aufführung, die wir nicht hören können. So verweist die Aufführungssituation des Radios, weil sie die sichtbare Quelle musikalischer Produktion verdeckt, auf die mit dem Hören stets miterscheinende Sphäre des Visuellen. Schon im Alltag entschlüsseln wir das undefinierbare Geräusch, indem wir es im Feld des Sichtbaren dingfest machen. Im Konzert wiederum verstehen wir einen Klang, indem wir die Geste der Hervorbringung von Menschen oder Maschinen sehen oder die räumliche Disposition von Klanggruppen im Aufführungssaal betrachten. In der Radiomusik müssen wir diese visuellen Gesten hinzuimaginieren und beleben auf diese Weise, durch unser audiovisuelles Hören, die "alte multimediale Konstellation in der Musik"13, von der Nicholas Cook gesprochen hat. Das Radio als das akustische Dispositiv par excellence verneint also den Mythos einer rein akustemischen Behauptung der Musik, die suggeriert, "dass Klang die eigentliche Essenz der Musik sei" $^{\prime 14}$, und liefert stattdessen eine Evidenz für die audiovisuelle Bestimmung von musikalischen Aufführungen. Dem Medium Radio Gehör zu schenken, thematisiert aber noch ein zweites Phänomen einer jeden musikalischen Aufführung, nämlich, dass sich diese stets zwischen Subjekt und Objekt der Wahrnehmung ereignet. Gerade die von Adorno an der Radiomusik kritisierte Disposition zur Privatheit der musikalischen Erfahrung, die im Extremfall eine immersive

13 Nicholas Cook, „Klang sehen, Körper hören. Glenn Gould spielt Weberns Variationen für Klavier", in: Musik und Geste: Theorien, Ansätze, Perspektiven, hrsg. von Katrin Eggers und Christian Grüny, München 2018, S. 71-88, hier S. 74.

14 Ebd., S. 72. 
Umschließung des Subjektes erwirkt, radikalisiert eine Tendenz ästhetischer Erfahrung, die in der sogenannten musikalischen Live-Aufführung seit jeher präsent war. Auch dort versenkt sich das Subjekt nicht nur in eine gleichsam objektivierte Musik, sondern es bespielt im Hören stets auch subjektive Erfahrungs- und Lebenswelten mit Musik. So artikuliert das Radio mit seinem Imperativ des „Hör' mir zu!" die Randbestimmungen dessen, was wir eine musikalische Aufführung nennen. Wenn wir dem Hören des Radios zuhören, dann wird es zum Erkenntnisinstrument, das unseren Begriff musikalischer Aufführung über die hörbare Musik hinaus, auf das Feld des Sichtbaren, und ebenso in die Leiber und Köpfe der hörenden Subjekte hinein ausdehnt.

Zitation: Magdalena Zorn, "Musik mit dem Radio hören: Über den Begriff der musikalischen Aufführung”, in: Freie Beiträge zur Jahrestagung der Gesellschaft für Musikforschung 2019, hrsg. von Nina Jaeschke und Rebecca Grotjahn (= Musikwissenschaft: Aktuelle Perspektiven. Bericht über die Jahrestagung der Gesellschaft für Musikforschung 2019 in Paderborn und Detmold, Bd. 1), Detmold 2020, S. 359-367, DOI: 10.25366/2020.77. 


\section{Abstract}

This article focuses on the phenomenon of listening to music via radio transmission. In an examination of linguistic findings and media archaeological observations, the specific performance characteristics of mediatized music are worked out using the example of a radio broadcast of a Beethoven symphony. The music-aesthetic and sociological essay "The Radio Symphony: An Experiment in Theory" (1941), written by Theodor W. Adorno during his stay in New York, is subjected to a re-reading. Although Adorno showed the full scope of his cultural conservatism in this essay, his thoughts nevertheless exemplify a function of technically mediated music reception that seems to be constitutive for the concept of musical performance as a whole.

\section{Kurzvita}

Magdalena Zorn, geboren 1984 in München, studierte Musikwissenschaft in Innsbruck, Freiburg i. B. und München. 2014 wurde sie mit der Arbeit Stockhausen unterwegs zu Wagner promoviert. Zu ihren jüngeren Buchveröffentlichungen zählt (Zu-)Hören interdisziplinär (Allitera 2018). Als Akademische Rätin auf Zeit am Institut für Musikwissenschaft der Ludwigs-MaximiliansUniversität München (LMU) leitet sie seit 2018 das DFG-Projekt "Die Licht-Räume der deutschen Nachkriegsavantgarde". 2020 hat sie sich an der LMU mit der Schrift "Was ihr hört: Werke, was sie durch uns gewesen sein werden" habilitiert. 


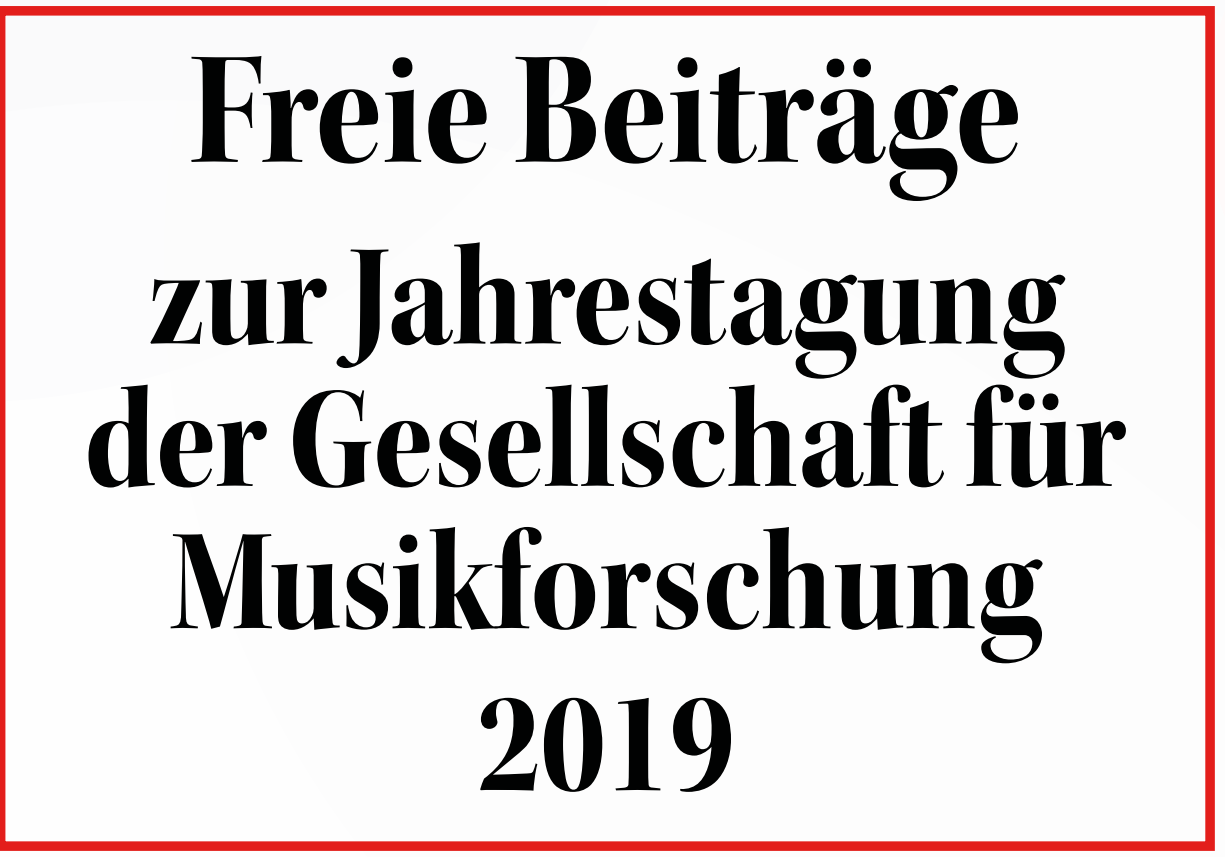

Herausgegeben von Nina Jaeschke und Rebecca Grotjahn

Musikwissenschaft: Aktuelle Perspektiven 1 
Freie Beiträge 


\section{Musikwissenschaft: Aktuelle Perspektiven}

Bericht über die Jahrestagung der Gesellschaft für Musikforschung 2019 in Paderborn und Detmold

Herausgegeben von Rebecca Grotjahn und Nina Jaeschke

Band 1 


\section{Freie Beiträge}

\section{zur Jahrestagung der Gesellschaft für Musikforschung 2019}

Herausgegeben von Nina Jaeschke und Rebecca Grotjahn

Detmold: Musikwissenschaftliches Seminar der Universität Paderborn und der Hochschule für Musik Detmold 2020 
DOI: $10.25366 / 2020.42$

Online-Version verfügbar unter der Lizenz: Urheberrecht 1.0, $<$ https://rightsstatements.org/page/InC/1.0/?language=de>

Bibliografische Information der Deutschen Nationalbibliothek

Die Deutsche Nationalbibliothek verzeichnet diese Publikation in der Deutschen Nationalbibliografie; detaillierte bibliografische Daten sind im Internet über http://dnb.d-nb.de abrufbar.

\section{Impressum}

Redaktion: Nina Jaeschke, Rebecca Grotjahn und Jonas Spieker Satz: Nina Jaeschke

(C) Musikwissenschaftliches Seminar der Universität Paderborn und der Hochschule für Musik Detmold 2020 


\section{INHALT}

Vorwort $\quad$ IX

Komponieren für das Radio: Akteure, Diskurse, Praktiken $\quad 1$

Musikwissenschaft - Feminismus - Kritik: Ein Generationenaustausch 6

\section{Stefan Alschner}

Der Wagner-Sänger Joseph Aloys Tichatschek - Vom Nachlass zum Netzwerk

\section{Alenka Barber-Kersovan}

Songs for the Goddess. Das popmusikalische Neo-Matriarchat zwischen Ethno-Beat,

erfundenen Traditionen und kommerzieller Vermarktung

Elias Berner, Julia Jaklin, Peter Provaznik, Matej Santi, Cornelia Szabó-Knotik

Musikgeschichte anders erzählen? Das Beispiel der 1970er in Österreich.

Musikhistoriographie in der Zeit der Digitalisierung

\section{Mauro Fosco Bertola}

„Ein Laut so klagevoll”. Lohengrin zwischen Richard Wagner und Salvatore Sciarrino

\section{Matthieu Cailliez}

Europäische Rezeption der Berliner Hofoper und Hofkapelle von 1842 bis 1849

\section{lacopo Cividini}

Zwischen klassischer Musikphilologie und angewandter Informatik:

Die Digitale Mozart-Edition (DME) der Stiftung Mozarteum Salzburg

\section{Marko Deisinger}

Fortschrittliche Technologie im Dienste eines Antimodernisten.

Heinrich Schenker und der österreichische Rundfunk

\section{Norbert Dubowy}

Vom Kritischen Bericht zur Kritischen Dokumentation am Beispiel der Digital-interaktiven Mozart-Edition

\section{Markus Engelhardt}

Musik zwischen Nation Building und Internationalität. Italien um 1900

\section{Maryam Haiawi}

Das Oratorium im Spannungsfeld der Konfessionen: 


\section{Judith I. Haug}

"Manch eine*r liegt, morgens noch trunken, im Rosengarten" - Rekonstruktionen

osmanischer Musikgeschichte in Gesangstextsammlungen

\section{Renate Koch}

Marcel Prawy und das erste Broadway-Musical im Österreich der Nachkriegszeit

Susanne Kogler, Julia Mair, Juliane Oberegger, Johanna Trummer

Erich Marckhl - Musikausbildung in der Steiermark nach 1945.

Brüche und Kontinuitäten

\section{Marie-Anne Kohl}

Die weinende Jury. "Geschlechtslose" Tränen bei globalen Musik-Castingshows?

\section{Fabian Kolb}

Tanztheater und filmische Ästhetik. Cineastische Einflüsse und Gestaltungsweisen in den Kompositionen für die Ballets Suédois 1920-1925

\section{Christian Lehmann}

Tempobezeichnungen von Julius Stockhausen für Die schöne Müllerin:

Ein Quellenfund

\section{Martin Link}

Signum et gens - Zur Gendersemiotik in Clara und Robert Schumanns Liederzyklus Liebesfrühling

\section{Livio Marcaletti}

„Strafspiel" und satirische Stilmittel in musikdramatischen Gattungen des frühen 18. Jahrhunderts

\section{Tobias Marx, Martin Lissner}

Thüringer Musikszene - Jugendmusikredaktionen als außerschulische musikbezogene Bildungskontexte

\section{Maho Naito}

Die Parallelität der Entstehungsprozesse der ersten beiden Symphonien Gustav

Mahlers: Instrumentation, Revision und Dirigierpraxis

\section{Elisa Novara}

Eine Schumann-Werkstatt? Zur Übertragbarkeit der Methoden vom Projekt 
Theodora Oancea, Joachim Pollmann, Jonas Spieker

Kollaborateure - Involvierte - Profiteure. Erarbeitung eines Online-Lexikons zur

Musik in der NS-Zeit

\section{Kiron Patka}

„Ich wollte eigentlich Sängerin werden." Berufsselbstbilder von Tontechniker*innen im Radio

\section{Siegwart Reichwald}

Die Leiden der jungen Clara: Das Klaviertrio Opus 17 als Ausdruck einer Neu-

Romantikerin

\section{Elisa Ringendahl}

Lied versus Oper - Pole musikalischer Gattungen bei Oscar Bie

\section{Benedikt Schubert}

Struktur und Exegese. Über Eigentümlichkeiten in der Arie "Des Vaters Stimme ließ sich hören" (BWV 7/4)

Uwe Seifert, Sebastian Klaßmann, Timo Varelmann, Nils Dahmen

Computational Thinking in der Musikwissenschaft: Jupyter Notebook als Umgebung

für Lehre und Forschung

\section{Yusuke Takamatsu}

Synthese als Modus der Prozessualität bei Schubert:

Sein spezifisches Wiederholungsprinzip im langsamen Satz

\section{Daniel Tiemeyer}

Johann Nepomuk Hummels Sonate in fis-Moll Op. 81 - Studien zu Entstehungs-

hintergrund, Rezeption und formalerStruktur

\section{Andrea van der Smissen}

Musikalische Innovation im Umfeld der Moderne und historischen Avantgarde in Ungarn

\section{Tim Ziemer, Holger Schultheis}

Psychoakustische Sonifikation zur Navigation in bildgeführter Chirurgie

\section{Magdalena Zorn}

Musik mit dem Radio hören: Über den Begriff der musikalischen Aufführung 
Gabriele Buschmeier in memoriam 


\section{Vorwort}

Die vorliegenden Bände dokumentieren die Jahrestagung der Gesellschaft für Musikforschung 2019. In den dreieinhalb Tagen vom 23. bis zum 26. September 2019 wurden in Paderborn und Detmold nicht weniger als 185 Beiträge präsentiert, verteilt auf diverse Symposien, Round tables, Freie Sektionen und Postersessions. Sie alle auf einen Nenner bringen zu wollen, ist ein Ding der Unmöglichkeit - und das ist gut so, ist es doch Ziel der Jahrestagungen, die große Vielfalt der Themen und Methoden des Faches Musikwissenschaft abzubilden. Um die thematische Vielfalt der freien Referate angemessen abbilden zu können und gleichzeitig den inhaltlichen Schwerpunkten der beiden hier publizierten Hauptsymposien ausreichend Raum bieten zu können, erscheinen diese in drei Bänden.

„Musikwissenschaft: Aktuelle Perspektiven": Der Titel der kleinen Reihe ist keine Verlegenheitslösung. Musikwissenschaft im Kontext der Digital Humanities; Musikwissenschaft und Feminismus; Musik und Medien; Musikalische Interpretation - schon die Felder, die von den vier Hauptsymposien bespielt wurden, wären noch vor wenigen Jahrzehnten allenfalls an der Peripherie das Faches zu finden gewesen. Sie entsprechen Arbeitsschwerpunkten der Lehrenden am Musikwissenschaftlichen Seminar der Universität Paderborn und der Hochschule für Musik Detmold, das die Tagung ausrichtete. Zugleich nehmen sie Bezug auf aktuelle Ereignisse und Entwicklungen. So erwuchs das von Andreas Münzmay und Joachim Veit organisierte Symposium „Brückenschläge - Informatik und Musikwissenschaft im Dialog" unmittelbar aus den Erfahrungen im Virtuellen Forschungsverbund Edirom (ViFE) und im fakultäten- und hochschulübergreifenden Zentrum Musik-Edition-Medien (ZenMEM). Der 200. Geburtstag von Clara Wieck/Schumann war der Anlass für das von Rebecca Grotjahn geleitete Symposium „Die Begleiterin - Clara Schumann, Lied und Liedinterpretation", das in enger Kooperation mit der Hochschule für Musik Detmold durchgeführt wurde. Das Hauptsymposium „Brückenschläge" wird in einem separaten Band publiziert (Bd. 3 der vorliegenden Reihe). Im Rahmen dieses Symposiums führte die von Stefanie Acquavella-Rauch geleitete Fachgruppe Digitale Musikwissenschaft eine Posterpräsentation durch, die von den Beiträger*innen erfreulicherweise zu kürzeren Texten umgearbeitet wurden, sodass sie hier ebenfalls, zusammen mit den Postern,

publiziert werden können. Hinzu kommen einige Beiträge, die bereits bei der Jahrestagung 2018 in Osnabrück präsentiert wurden. Auch das Hauptsymposium "Die Begleiterin" wird in einem eigenen Band (Bd. 2) publiziert. Die Beiträge zu den beiden anderen Hauptsymposien hingegen werden an anderen Orten veröffentlicht; in Band 1 („Freie Beiträge zur Jahrestagung der Gesellschaft für Musikforschung 2019") der vorliegenden Publikation finden sich jedoch Einführungen und Abstracts. Das Symposium „Komponieren für das Radio" unter Leitung von Antje Tumat und Camilla Bork (Katholieke Universiteit Leuven) behandelte Einflüsse des Mediums auf Kompositionsprozesse sowie durch radiophone Kompositionen bzw. radiophonen Klang ausgelöste Diskurse. Sarah Schauberger und Cornelia Bartsch (Universität Oldenburg) nahmen das 25-jährige Jubiläum der Fachgruppe Frauen- und Genderstudien zum Anlass für einen Generationenaustausch zum Thema "Musikwissenschaft - Feminismus - Kritik": Was wa- 
ren vor einem Vierteljahrhundert Inhalte und Aufgaben einer feministischen Musikwissenschaft und wie kann sich diese heute positionieren?

Bewusst haben wir im Tagungsbericht auf inhaltliche Eingriffe in die Beiträge verzichtet. ${ }^{1}$ Das gilt besonders für die Freien Referate: Es galt, den Charakter der Jahrestagung als Forum für ,freie', d. h. innovative und auch experimentelle Gedanken zu wahren. Einige Kolleg*innen, die die Tagung mit Vorträgen und Posterpräsentationen bereichert hatten, haben sich gegen eine Publikation im vorliegenden Band entschieden - sei es, weil sie eine Möglichkeit fanden, ihre Beiträge in einem inhaltlich passenderen Rahmen zu veröffentlichen, sei es, weil ihre Überlegungen in ihre entstehenden Qualifikationsschriften fließen sollen, oder sei es, weil sie von den Autor*innen in der vorgetragenen Form zunächst verworfen wurden. Auch damit erfüllt eine Freie-Referate-Sektion ihren Zweck: Die Diskussionen mit der versammelten Fach-Öffentlichkeit sollen dabei helfen, Gedanken weiterzuentwickeln und zu verändern. In diesem Sinne sei allen Beteiligten - den Autor*innen, den nichtpublizierenden Referent*innen und den Mit-Diskutant*innen - ganz herzlich gedankt für ihr Mitwirken bei der Tagung.

Unser herzlicher Dank gilt einer Reihe weiterer Personen, die zum Gelingen dieser drei Bände beigetragen haben. Hier ist besonders Jonas Spieker zu nennen, der uns tatkräftig bei der Redaktion geholfen hat. Andrea Hammes (SLUB Dresden) sei herzlich für die Aufnahme unseres Bandes auf musiconn.publish gedankt - wir freuen uns, damit unsererseits zur Etablierung dieser innovativen Publikationsplattform beizutragen.

Erneut möchten wir an dieser Stelle allen Menschen danken, die uns bei der Organisation, Ausrichtung und Finanzierung der Tagung selbst unterstützt haben: der Präsidentin der Universität Paderborn, Prof. Dr. Birgitt Riegraf, dem Rektor der Hochschule für Musik Detmold, Prof. Dr. Thomas Grosse, den Kolleginnen und Kollegen der beiden beteiligten Hochschulen, dem Vorstand der Gesellschaft für Musikforschung, der Universitätsgesellschaft Paderborn und allen Sponsoren. Besonders dankbar sind wir den Mitarbeiter*innen und den studentischen bzw. wissenschaftlichen Hilfskräften des Musikwissenschaftlichen Seminars, die bei der Vorbereitung und Ausrichtung der Tagung immensen Einsatz zeigten - stellvertretend sei an dieser Stelle Johanna Imm erwähnt, die zusammen mit Nina Jaeschke das Herz des Organisationsteams bildete.

Wir widmen diese Reihe Dr. Gabriele Buschmeier, dem langjährigen Vorstandsmitglied der Gesellschaft für Musikforschung, die kurz vor der Publikation dieses Bandes unerwartet verstarb.

Detmold, im September 2020

Rebecca Grotjahn und Nina Jaeschke

Zitation: Rebecca Grotjahn und Nina Jaeschke, „Vorwort”, in: Freie Beiträge zur Jahrestagung der Gesellschaft für Musikforschung 2019, hrsg. von Nina Jaeschke und Rebecca Grotjahn (= Musikwissenschaft: Aktuelle Perspektiven. Bericht über die Jahrestagung der Gesellschaft für Musikforschung 2019 in Paderborn und Detmold, Bd. 1), Detmold 2020, S. IX-X, DOI: 10.25366/2020.43.

1 Freigestellt war den Autor*innen auch, ob sie sich für eine gendersensible Sprache entscheiden bzw. welche Form des Genderns sie bevorzugen. 